DOI: $10.17234 / S R A Z .65 .11$

UDK: 81'34-05 Guberina, P.

Original scientific paper

Reçu le 24 octobre 2020

Accepté pour la publication le 25 novembre 2020

\title{
La linguistique de la parole : de Saussure à Guberina et au-delà
}

Bogdanka Pavelin Lešić

Faculté de philosophie et lettres, Université de Zagreb bpavelin@ffzg.hr

La linguistique de la parole, annoncée par Saussure, implique l'étude scientifique de la langue en tant que partie intégrante de l'être humain et de sa parole. Ce choix rend possible l'application transdisciplinaire de la théorie linguistique de Guberina à des domaines allant de l'enseignement des langues étrangères jusqu'à la rééducation de l'audition et de la parole. En utilisant le syntagme de langue parlée, Guberina insiste sur l'unité de la langue et de la parole au sein du langage et reprend le concept d'affectivité établi par Ch. Bally. Sans être humain il n'y a ni discours ni activité langagière authentiques, c'est pourquoi la parole et la langue parlée se trouvent au centre de l'étude linguistique guberinienne et l'être communiquant en constitue le fondement.

Mots clefs : Valeurs de la langue parlée, langage, affectivité, linguistique de la parole, être communiquant

\section{Introduction}

P. Guberina (1913-2005) est l'un des premiers linguistes à avoir explicitement positionné toute sa recherche scientifique dans le domaine de la linguistique de la parole. D'un côté, Guberina s'est imprégné de la théorie du CLG (1916) de Saussure et de la stylistique linguistique de Bally. De l'autre, il a élaboré sa propre approche théorique et méthodologique du langage dans le domaine de la linguistique de la parole, en transférant la notion du système de la langue au composé complexe et dynamique du langage.

\section{Les repères théoriques saussuriens}

Le CLG fut le point de départ des recherches de Guberina. Il l'a lu au début des années trente du vingtième siècle, en tant qu'étudiant au Département d'études romanes à la Faculté de philosophie et lettres de Zagreb. La linguistique de la parole n'y est qu'envisagée ([1916] 1978 : 10). Cependant, dès les années 1930, Guberina oriente ses recherches dans le domaine de la linguistique de la parole, annoncée par Saussure dans le Chapitre IV de l'Introduction intitulé Linguistique de la langue et linguistique de la parole. 
Guberina a d'emblée adopté la conception structurale du langage : si une modification se produit pour un composant à un niveau particulier, la structure de l'ensemble du système est également modifiée. Nonobstant, il ne cantonne pas ses recherches au système linguistique indépendant de la situation et des êtres communiquant. Il préfère aborder la langue en tant que partie de l'intégralité du langage. La cohésion du macro-système langagier découle du fonctionnement de l'ensemble véhiculant du sens dans la situation communicationnelle.

Guberina (1938) utilise comme point de départ le constat saussurien de la primauté de l'oral sur l'écrit. La langue parlée représente la double essence du langage humain. C'est un ensemble à la fois linéaire ou segmental et synthétique ou suprasegmental. Les êtres communiquant, sujet parlant et sujet interprétant, imprègnent le texte écrit de toutes les marques de l'oralité, ce qui amène Guberina à constater (2003 : 158) : Il n'y a pas de langue écrite ; il existe seulement la langue qui peut être parlée ou écrite. La langue écrite est toujours une transposition de la langue parlée, et il faut bien composer pour que le lecteur devine les valeurs de la langue parlée ([1939] $1954: 62,227)$.

Les titres des ouvrages Valeur logique et valeur stylistique des propositions complexes (1939) et La solidarité des éléments du langage (1952) reflètent l'adhésion de Guberina à la terminologie et à la pensée saussuriennes. Nonobstant, la lecture de Saussure par Guberina s'est avérée sous certains aspects en rupture avec la lecture structuraliste dominante. Le problème du tout dans l'expression langagière demeure essentiel pour Guberina. Cependant, il s'intéresse au problème du tout au-delà de la langue, ses études traitent du problème du tout dans le langage en observant la parole en tant que réalisation de la langue et d'autres ensembles de l'expression langagière.

La langue demeure la force centripète qui fait converger les éléments langagiers hétérogènes vers une unité structuro-globale, mais elle n'est plus le seul principe de classification : le principe central c'est l'homme. L'être humain s'exprime et développe sa pensée par le biais du langage et son affectivité dicte le choix des moyens d'expression langagière dans une situation concrète. La façon dont les moyens langagiers plurimodaux vont se développer et se réaliser dépend de la situation. La structure langagière selon Guberina fonctionne comme un ensemble situationnel conçu sur la base des rapports réciproques et permanents entre société et individu. La structure langagière se réalisant dans la parole, il s'agit évidemment d'un phénomène dynamique. C'est pourquoi Guberina finit par opter pour le terme de structuration (2003 [1976] : 257). Compte tenu de la structuration complexe des faits de langage, la linguistique de la parole s' ouvre en permanence à la fois à la recherche fondamentale en langue et parole et aux nouveaux types d'application pratique.

Guberina a reconnu chez Saussure l'importance de la chaîne qui relie l'émission du langage à sa réception, la dernière se transformant d'emblée en émission et vice versa, ce qui implique le caractère dialogal de l'expression langagière : C'est un fait que le langage se présente comme un dialogue continu (2003 : 259).

Saussure insiste sur le caractère uniquement individuel de la parole alors que chez Guberina, la parole est un phénomène à la fois individuel et dialogal, donc social, sinon l'interprétation en serait impossible. Guberina constate que la parole est un ensemble situationnel exprimé par un système formel propre à la structure 
de la parole (2003 : 257). Pour Guberina, Saussure a indirectement introduit l'homme dans le système par sa conception de l'étude de langue en synchronie et en diachronie, par l'axe syntagmatique et associatif, par l'approche externe et interne de la langue, qui implique le positionnement de la langue par rapport à l'être humain et non l'inverse. Guberina a accepté aussitôt cet héritage théorique et il a tracé sa voie dans la direction de la linguistique de la parole, alors que la théorie de Saussure était encore en train d'être découverte, interprétée et adoptée. Guberina étudie la langue parlée comme partie vivante de l'être communiquant qui utilise son corps, son cerveau et ses sentiments de manière créative pour communiquer à travers des moyens d'expression plurimodaux. Il conclut :

Les faits de langue se réalisent de deux manières : l'un se rattache à l'oreille et l'autre à l'cil. Voilà pourquoi nous divisons les valeurs de la langue parlée en deux groupes : 1) les valeurs acoustiques, 2) les valeurs visuelles. [...] Appartiennent aux valeurs acoustiques : a) l'intonation (la mélodie) - b) l'intensité - c) le temps de la phrase - d) les pauses (le silence) ; aux valeurs visuelles: a) la mimique ${ }^{1}-b$ ) les mouvements $-c$ ) la situation ([1939] 1954 : 59-60).

\section{Influence de Bally sur Guberina}

Les termes logique et stylistique dans le titre du livre Valeur logique et valeur stylistique des propositions complexes (1939) relèvent de l'influence de Bally sur Guberina. D'un côté la logique est le point de départ pour délimiter et identifier les faits de langage, de l'autre la présence du sujet communiquant dans l'énoncé détermine la véritable valeur de l'expression. ${ }^{2} \mathrm{Ch}$. Bally s'intéresse à la subjectivité dans la langue tandis que P. Guberina observe la subjectivité dans le langage. Si le sujet parlant est le point central de la stylistique linguistique de Bally, le point central de la linguistique de la parole de Guberina est le sujet communiquant. Tous deux valorisent la notion d'affectivité. ${ }^{3}$ La stylistique de Bally consiste à étudier le contenu affectif des faits d'expression linguistique. Bally met en œuvre le procédé de délimitation par lequel on délimite les unités de pensée, non les unités lexicales. Ainsi reconnaît-il l'aspect pluridimensionnel du contenu (intellectuel, affectif, effet par évocation), le rôle du contexte (1936 : 87) et l'aspect plurimodal de la parole. Bally constate que l'on ne peut prononcer des mots ayant un sens sans que la pensée elle-même porte une intonation, identique à celle des mots par lesquels nous l'exprimerions (ib. : 94). Il reconnaît aussi le rôle de la situation ou de la mise en scène de la langue parlée et les jeux de physionomie, les gestes, tous les mouvements du corps comportant une valeur conventionnelle et symbolique (ib. : 92).

La démarche de Guberina consiste à examiner la structure d'un énoncé complexe, sa réalisation contextuelle et situationnelle globale. Une phrase ne

1 La mimique et les mouvements du corps sont appelés en général gestes. Le terme de mimique renvoie aux mouvements du visage.

2 Le dictum et le modus.

3 Pour Bally l'affectivité est la marque extérieure de l'intérêt personnel que nous prenons à la réalité (1952:75). 
peut être le point de départ d'une description linguistique si elle est considérée uniquement comme unité formelle privée de son sens situationnel. Le point de départ de l'analyse doit être le sens, c'est-à-dire le contenu logique et stylistique (affectif, subjectif) de l'énoncé. L'énoncé ne dépend pas du nombre de mots ou du type de mot utilisé. L'intonation et les mouvements corporels issus de la situation sont à la base de chaque réalisation en langue parlée. Toute phrase fait partie d'un énoncé-discours en tant que résultat d'une situation concrète, d'une réaction concrète de l'être communiquant qui n'est jamais indifférent à ce qu'il fait (1954 [1939] : 32). Le sens de l'expression langagière n'est pas réductible au seul contenu intellectuel, mais comprend également le contenu affectif qui, ensemble font un seul sens et une seule qualité : la valeur affective de l'expression et aussi objective, plus objective que sa valeur objective même (ib. : 29).

\section{Pour une logique du langage qui prend en compte l'être communiquant, créateur du sens}

La prise en compte des êtres communiquant dans la linguistique de la parole implique un changement de mode de raisonnement. Les grammaires traditionnelles décrivaient la langue en décrivant les parties du discours, avec les taxonomies héritées du grec et du latin, reprises par la Grammaire de Port-Royal (2000 [1660]), suivies par les grammaires qui ont succédé à Port-Royal. Cette approche repose sur la logique formelle ou la logique de la logique (Guberina 1957). Ni les êtres communiquant ni l'usage ordinaire de la langue parlée dans une situation particulière ne sont pris en compte dans les études linguistiques et grammaticales fondées sur la logique traditionnelle. Tout au long du XXe siècle, les études linguistiques ont révélé des points faibles de la logique pratiquée par les théories traditionnelles de la grammaire. La logique traditionnelle, ou la logique de la logique, n'a pas réussi à révéler les mécanismes de structuration dynamique du langage.

Dans son livre La solidarité des éléments du langage (1952), et dans son article La logique de la logique et la logique du langage (1957), Guberina constate les limites de la description de la langue à partir de la logique formelle où l'être communiquant est absent dans la formule S - (donne) P.

La logique du langage proposée par Guberina est censée permettre d'analyser la manière dont l'homme façonne sa pensée et son expression par le biais du langage. Guberina constate que l'être humain est à la fois le manifestans et la manifestation, ${ }^{4}$ la cause et l'effet, le créateur et le phénomène naturel et social.

M. Masterman (2005 [1963]), pionnière dans le domaine de la linguistique computationnelle et de la traduction automatique, et précurseure de la linguistique cognitive, a reconnu l'importance de la logique du langage proposée par Guberina. Elle utilise le syntagme hypothèse de Guberina pour faire référence à une formule unique de progression sémantique à la base de toute communication

4 ms et $m a$

5 ... there exists a single formula for semantic progression at the basis of all human communication (2005 : 227). 
humaine. ${ }^{5}$ Guberina introduit le carré dans l'équation $\mathrm{S}^{2}=($ donne $) \mathrm{P}^{2} \mathrm{ou}$, selon sa propre terminologie, $\mathrm{ms}^{2}=\mathrm{ma}^{2}$. Le carré de l'équation désigne le rôle de l'homme qui est présent dans toute énonciation, l'homme-agens crée de nouveaux concepts sur la base de concepts de départ. Les unités de langage, de forme apparemment linéaire, sont en fait des sous-ensembles solidaires constitutifs des unités de pensée. Les manifestans se manifestent dans une certaine manifestation (ma). Le manifestans (ms) manifesté dans la manifestation (ma), l'un en fusion avec l'autre, devient alors une nouvelle réalité, un nouveau manifestans manifesté dans une nouvelle manifestation ... sa manifestation s'élargit et devient un nouveau manifestans (> qui se manifeste au moyen de, qui ensemble donne ms, un nouveau manifestans) : $m s^{2}>m a^{2} » \underline{m s}^{2}>m a^{2} » \underline{m s^{2}>m a^{2}} \ldots$

Dans toute expression linguistique découlant d'un discours, sous forme orale ou écrite, nous assistons toujours à des manifestans manifestés au carré, où le carré est formé par le manifestans manifesté en tant qu'homme communiquant et où le ms-ma de base est donné par le concept connu jusqu'à un certain degré et par la nature elle-même. L'être humain peut, par son cerveau, observer, supposer, réfléchir sur des manifestations encore inconnues, non manifestées d'un manifestans. L'être communiquant est également susceptible de réaliser de nouveaux manifestans et de nouvelles manifestations en étant créateur perpétuel.

Tout se manifeste sous forme phénoménale : le monde réel, la vie humaine ainsi que la pensée et l'expression langagière. Le langage se déroule aussi sous un aspect phénoménal ${ }^{6}$ car existant pour les hommes et par les hommes, la langue parlée reçoit tous les éléments que l'homme peut lui fournir, elle est pleine de tous les signes de la vie humaine (Guberina 1954 : 59, 1957 : 17).

En fait, l'activité linguistique n'existe pas en dehors de l'individu, de la pensée humaine et de l'ensemble $m s^{2}>m a^{2}$ La logique du langage de Guberina prend en compte la cohérence qui sous-tend l'unité de l'usage du langage, une cohérence qui n'a pas été capturée par la logique formelle ou par la seule linguistique de la langue.

\section{En guise de conclusion}

Selon Guberina, les linguistes peuvent et doivent aborder tous les problèmes de la complexité de l'expression humaine en reliant l'expression langagière à la pensée, à l'affectivité, aux valeurs de la langue parlée et à la situation. La pensée théorique de Guberina constitue un repère scientifique précieux pour aborder la problématique du langage humain et de son expression. N'excluant pas la linguistique de la langue, la linguistique de la parole finit par rassembler de nombreux domaines des linguistiques contemporaines (énonciatifs, cognitivistes ...). Les nombreux écrits de Guberina, malgré l'apparente diversité des sujets allant de l'enseignement des langues jusqu'à la rééducation orthophonique et au-delà, découlent d'une pensée profonde, complexe et visionnaire sur le langage humain dans laquelle tout est d'une manière ou d'une autre lié dans un système théorique cohérent et ouvert aux nouveaux développements.

6 Pojavni lik jezika (1952 : 10) 


\section{Références bibliographiques :}

Bally, Charles 1936 [1909]. Traité de stylistique française, premier volume, Paris : Klincksieck.

Bally, Charles 1952 [1913]. Le Langage et la vie, Genève : Droz.

CLG $=$ Saussure, Ferdinand 1978 [1916]. Cours de linguistique générale, Paris : Payot.

Guberina, Petar (1938). Govorni i pisani jezik/La langue parlée et la langue écrite, in Hrvatski jezik 1/6-7, 114-124.

Guberina, Petar (1957) La logique de la logique et la logique du langage, in Studia Romanica Zagrabiensia II/3, pp. 13-30.

Guberina, Petar (1952) Povezanost jezičnih elemenata/La Solidarité des éléments du langage, Zagreb : Matica hrvatska.

Guberina, Petar 1954 [1939]. Valeur logique et valeur stylistique des propositions complexes, Zagreb : Epoha.

Guberina, Petar (2003). Rétrospection, éd. C. Roberge, Zagreb : ArtTrezor.

Lancelot, Claude/Arnauld, Antoine (2000 [1660]). Grammaire générale et raisonnée de Port-Royal /Opća obrazložbena gramatika Port-Royal, Zagreb : IHJJ, ur. A. Kovačec, komentari V. Vinje.

Masterman, Margaret (1963) Commentary on the Guberina hypothesis, Methodos, XV in : (2005) Language, Cohesion and Form [éd. Y. Wilks], Cambridge : Cambridge University Press, 227-252.

Masterman, Margaret (1968) Semantic Algorithms, CLRU memo in : (2005) Language, Cohesion and Form, éd. Yorick Wilks, Cambridge : Cambridge University Press, 253-280.

\section{Lingvistika govora, od Saussurea do Guberine i dalje}

P. Guberina (1913-2005) je među prvim lingvistima svoj opus izrijekom smjestio u lingvistiku govora. Već iz naslova Valeur logique et valeur stylistique des propositions complexes (1939) i Povezanost jezičnih elemenata (1952) iščitavamo utjecaj Saussurea na Guberinu: u jeziku i jezičnoj djelatnosti sve je povezano i zasnovano na vrijednostima koje proizlaze iz međusobne povezanosti sastavnih dijelova cjeline. Saussure u Tečaju opće lingvistike (1916) poziva na razvijanje lingvistike jezika - linguistique de la langue - i najavljuje razvoj lingvistike govora - linguistique de la parole. Govorni jezik je u središtu Guberininog lingvističkog proučavanja, a ljudsko biće je pritom polazišna osnovica jer bez čovjeka nema ni autentične govorno-jezične djelatnosti ni diskursa. Od Ballyja Guberina preuzima pojam afektivnosti. Čovjek iskazuje i razvija misaonost govorno-jezičnim izrazom, a njegova afektivnost diktira odabir sredstava tog izraza. Guberina smatra da jezikoslovci mogu i moraju zaći u sve probleme složenog ljudskog izraza povezivanjem govorno-jezične djelatnosti s mišlju, afektivnosti, vrednotama govornog jezika i situacijom.

Ključne riječi: Vrednote govornog jezika, jezična djelatnost, afektivnost, lingvistika govora, komunicirajuće biće 